\title{
Indirect Assessment of Small Hydrophobic Ligand Binding to a Model Protein Using a Combination of ESI MS and HDX/ESI MS
}

\author{
Hui Xiao and Igor A. Kaltashov \\ Department of Chemistry, University of Massachusetts, Amherst, Massachusetts, USA \\ Stephen J. Eyles \\ Department of Polymer Science and Engineering, University of Massachusetts, Amherst, Massachusetts, USA
}

Direct mass spectrometric characterization of interactions between proteins and small hydrophobic ligands often poses a serious problem due to the complex instability in the gas phase. We have developed a method that probes the efficacy of ligand-protein interactions indirectly by monitoring changes in protein flexibility. The latter is assessed quantitatively using a combination of charge state distribution analysis and amide hydrogen exchange under both native and mildly denaturing conditions. The method was used to evaluate binding of a model protein cellular retinoic acid binding protein I to its natural ligand all-trans retinoic acid (RA), isomers 13-cis- and 9-cis-RA, and retinol, yielding the following order of ligand affinities: All-trans RA > 9-cis RA > 13-cis RA, with no detectable binding of retinol. This order is in agreement with the results of earlier fluorimetric titration studies. Furthermore, binding energy of the protein to each of retinoic acid isomers was determined based on the measured hydrogen exchange kinetics data acquired under native conditions. (J Am Soc Mass Spectrom 2003, 14, 506-515) () 2003 American Society for Mass Spectrometry

I n the past decade, mass spectrometry has come to play a major role in characterization of protein structure, dynamics and function [1-3]. Mass spectrometry is particularly useful to study protein-ligand interactions, owing to its ability to determine ligand composition and binding stoichiometry by direct mass measurement of the protein-ligand complex following its desorption from solution by means of electrospray ionization (ESI) [1]. Furthermore, charge state distributions in the ESI spectra of folded and unfolded proteins provide a useful tool to study protein conformation at low resolution [4, 5] and its relationship to ligand binding [6]. Proteins under native conditions show narrow charge state distributions in mass spectra with a small number of charges, whereas those under denaturing conditions tend to have broader charge state distribution with a higher number of charges. Thus, one can monitor protein conformations present in solution at equilibrium, and also kinetically during folding with time-resolved ESI-MS [7, 8].

Mass spectrometry is also useful for characterizing the interface regions within multimeric proteins. This is usually achieved by applying either chemical cross-

Published online April 2, 2003

Address reprint requests to Dr. I. A. Kaltashov, Department of Chemistry, Lederle Graduate Research Center no. 701, 710 North Pleasant Street, University of Massachusetts, Amherst, MA 01003, USA. E-mail: kaltashov@chem.umass.edu linking [9] or amide hydrogen/deuterium exchange (HDX) [2]. HDX/ESI-MS (or HDX/MALDI-MS) measures the change of deuterium content by monitoring the mass change as a function of exchange time. Exchange rates of backbone amide protons are strongly dependent on their environments: HDX is affected by the involvement in hydrogen bonding and solvent accessibility [10].

Although HDX has been successfully applied to investigate protein-protein complexes with large interfaces [11], it is not readily possible to use this approach for direct mapping of small ligand binding; when the ligand is small there is a lack of efficient shielding from solvent at the intermolecular interface. Small hydrophobic ligands present a particularly difficult case, as the protein-ligand interaction in this case does not involve formation of hydrogen bonds that can be readily detected in HDX-MS experiments. Another experimental challenge often posed by small hydrophobic ligands is their facile dissociation from proteins in the gas phase. Indeed, the removal of solvent molecules from the protein-ligand complex environment (upon its desorption to the gas phase) reduces the entropy-driven hydrophobic interactions to short-range van der Waals interactions, while long-range (electrostatic) interactions become the major determinant of the protein ion structure [12]. As a result, even very mild desorption conditions often cause partial or complete complex 

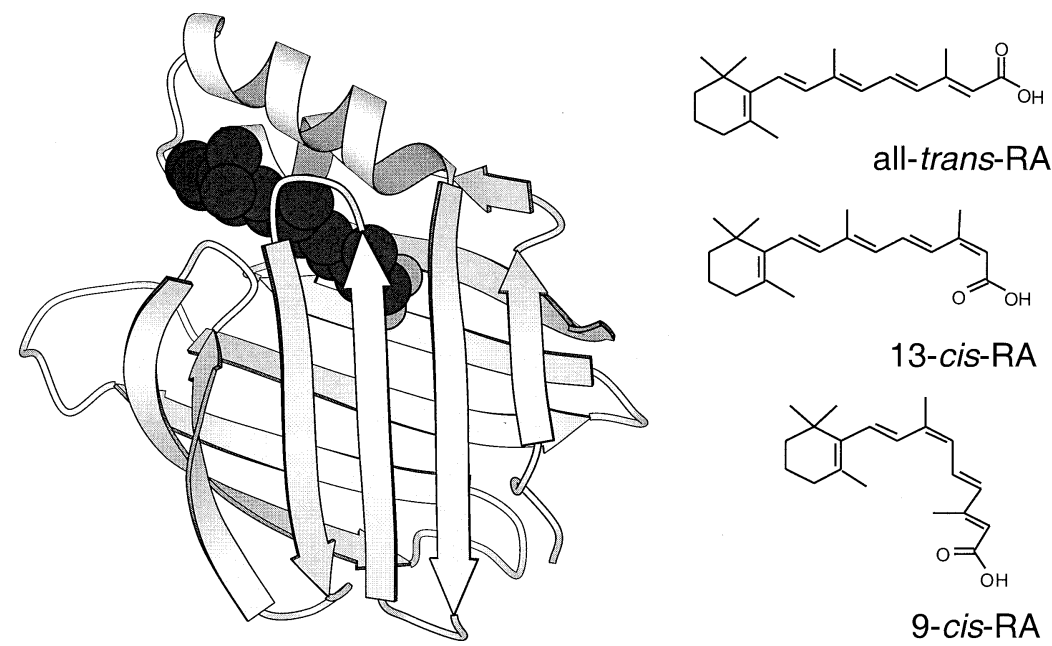

Figure 1. Ribbon diagram of cellular retinoic aicd binding protein I complexed with all-trans-retinoic acid (PDB 1CBR) and the chemical structures of the three isomers of retinoic acid studied in this work.

dissociation in the gas phase, thus compromising the quality of ESI-MS based stoichiometric assignments [13].

In this paper we evaluate indirect mass spectrometric methods to characterize binding of small hydrophobic retinoid ligands to a model protein, cellular retinoic acid binding protein I (CRABP I). CRABP I is a member of a family of small soluble intracellular proteins that bind hydrophobic ligands such as fatty acids, lipids, and retinoids [14]. This $15 \mathrm{kDa}$ protein contains 136 residues which form two 5 -stranded $\beta$-sheets. The first two strands are connected by a helix-turn-helix motif, and all of others by reverse turns. The two $\beta$-sheets are packed orthogonally to form a solvent-filled $\beta$-barrel.

The ligand-binding pocket, which physiologically accommodates all-trans retinoic acid (RA) is located inside the barrel (Figure 1). Importantly, the native ligand all-trans retinoic acid (ATRA), binds CRABP I predominantly by hydrophobic interactions, as the ligand forms ten contacts with non-polar side chains and only one salt bridge [15]. The overall contribution to the free energy of the protein-ligand complex formation in solution was estimated to be in the range of 5-6 $\mathrm{kcal} / \mathrm{mol}$ [16]. The ligand binding/release mechanism by CRABP I has not been clearly established. A "portal" model has been postulated, which invokes the notion of a highly dynamic segment within the protein that serves as an opening, thus allowing the entry of ligand into the cavity [17, 18]. In CRABP I this portal region constitutes the helix-turn-helix motif and two flanking $\beta$-hairpins. Reduced dynamics within this region in the holo-form of the protein effectively inhibits dissociation of the complex in solution [19].

We have previously demonstrated that such changes in protein dynamics can be probed using a variety of ESI-MS based strategies [20, 21]. Therefore, protein backbone dynamics can serve as a reliable, although indirect, gauge of complex formation in solution. Using this approach, one can probe complex formation in solution under near native conditions and at physiological concentrations. In addition to ATRA, we have also included in our studies a set of non-cognate (but natural) retinoid ligands, retinoic acid isomers (9-cis-RA and 13-cis-RA), and retinol. The hydrophobic skeletons of the former two are altered sufficiently to introduce significant disruption to the network of "native" hydrophobic contacts, thus reducing stability of the complex as demonstrated by fluorescence titration [22]. Retinol, the fourth ligand used in our studies, does not form a complex with CRABP I [23]. We have been able to rank all four ligands according to their CRABP I affinity by monitoring protein dynamics measured by ESI-MS. This mass spectrometry-based method may be a reasonable alternative to NMR when high protein concentrations can not be achieved and/or high molecular weight of the protein places it beyond the reach of high-resolution NMR measurements. The method may find uses in aiding the design of therapeutically active ATRA analogs with enhanced binding to CRABP I [24] or other retinoid carriers [25].

\section{Experimental}

\section{Materials}

All retinoids used in this work were purchased from Sigma-Aldrich (St. Louis, MO). CRABP I samples were generously provided by Professor L.M. Gierasch (University of Massachusetts). The protein sample was deuterated by replacing all labile hydrogen atoms with deuterium in several cycles of dissolution in $\mathrm{D}_{2} \mathrm{O}$ and acetic- $\mathrm{d}_{4}$ buffer solution, followed by lyophilization.

\section{Methods}

For the exchange experiments, dry protein was dissolved to a concentration of $\sim 1 \mathrm{mg} / \mathrm{ml}$ with $\mathrm{D}_{2} \mathrm{O}$ and acetic- $\mathrm{d}_{4}$ buffer solution. Back-exchange was initiated 
by diluting the protein solution 1:50 in a desired $\mathrm{pH}$ level protiated buffer solution $(10 \mathrm{mM}$ ammonium acetate). All retinoids were initially dissolved at $3 \mathrm{mM}$ concentration in methanol. Small aliquots of such solutions [typically 1:500 (vol:vol) dilution] were added to a $1.2 \mu \mathrm{M}$ protein solution at $\mathrm{pH} 7$ to maintain the methanol level below $0.5 \%$. The final molar ratio of RA to protein was thus 5:1, unless specified otherwise. The protein concentration was calculated from UV absorption at $280 \mathrm{~nm}$ using extinction coefficient $20,970 \mathrm{M}^{-1}$ $\mathrm{cm}^{-1}$ [26]. The concentrations of retinoid stock solutions were measured using an extinction coefficient of 45,000 $\mathrm{M}^{-1} \mathrm{~cm}^{-1}$ at $350 \mathrm{~nm}$ [27]. Composition of each retinoid stock solution was monitored by HPLC (C18 column) prior to each experiment to verify absence of isomerization [28].

ESI-MS data were acquired using a JMS-700 MStation (JEOL, Tokyo, Japan) magnetic sector mass spectrometer. Samples were continuously infused into the electrospray ionization source at a $5 \mu \mathrm{l} / \mathrm{min}$ flow rate with an aluminum foil protected syringe (to avoid photo-isomerization of retinoids in solution). Mass spectra were acquired by scanning the magnet at a 5 $\mathrm{s} /$ decade rate. Each recorded spectrum was an average of $30-50$ scans.

\section{Results and Discussions}

\section{Evaluation of the Protein-Ligand Complex Stability in the Gas Phase}

In line with expectations, the ATRA-CRABP I complex was found to be rather unstable in the gas phase, owing to the mostly hydrophobic character of the interaction. The relative ionic signal of the ligand-bound protein was found to be highly sensitive to ESI source conditions (orifice/ring lens voltage and desolvating plate/ orifice temperature). At high voltage and intermediate temperature $\left(180 / 300 \mathrm{~V}, 120 / 80^{\circ} \mathrm{C}\right)$, only ions corresponding to the mass of the apo-protein were observed (Figure 2a). The protein-ligand complex ions were detected only following simultaneous reduction of voltage and temperature (Figure 2b, c). Under the mildest possible desolvating conditions (orifice/ring lens voltage $0 / 60 \mathrm{~V}$ and desolvating plate/orifice temperature $80 / 60^{\circ} \mathrm{C}$ ), complex ion peaks of only 1:1 stoichiometry were observed (Figure 2d).

Interestingly, apo-protein ions are still observed in the mass spectrum of an equimolar mixture of ATRA and CRABP I, even under the mildest conditions applied. Further reduction of the source temperature to ambient resulted in much lower $\mathrm{S} / \mathrm{N}$ ratio, but did not alter the intensity ratio of the apo- and holo-protein ions (ca. 98\%). The dissociation constant measured by fluorescence titration $\left(\mathrm{K}_{\mathrm{d}}=0.4 \mathrm{nM}\right.$ [22]) suggests that the fraction of free (unbound) ligand in solution is less than $0.01 \%$. It is clear, therefore, that dissociation of a significant proportion of the complex must occur in the gas phase. Upon increasing of the molar ratio stepwise from

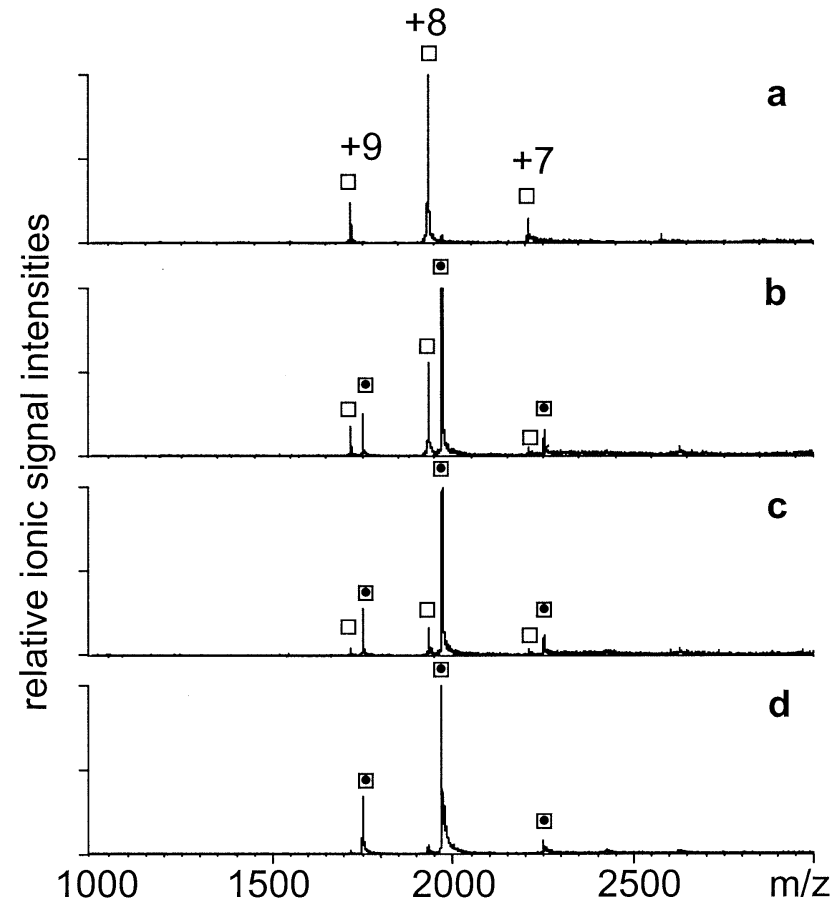

Figure 2. ESI MS: Stability of the CRABP-RA complex ions in the gas phase as a function of orifice $1 /$ ring lens voltage $\left(\mathrm{V}_{\mathrm{O}-\mathrm{L}}\right)$ and desolvating plate/orifice 1 temperature $\left(\mathrm{T}_{\mathrm{D}}\right): \mathrm{V}_{\mathrm{O}-\mathrm{L}}=120 \mathrm{~V}, \mathrm{~T}_{\mathrm{D}}=$ $80{ }^{\circ} \mathrm{C}(\mathbf{a}) ; \mathrm{V}_{\mathrm{O}-\mathrm{L}}=100 \mathrm{~V}, \mathrm{~T}_{\mathrm{D}}=80^{\circ} \mathrm{C}(\mathbf{b}) ; \mathrm{V}_{\mathrm{O}-\mathrm{L}}=60 \mathrm{~V}, \mathrm{~T}_{\mathrm{D}}=80^{\circ} \mathrm{C}$ (c); $\mathrm{V}_{\mathrm{O}-\mathrm{L}}=60 \mathrm{~V}, \mathrm{~T}_{\mathrm{D}}=60^{\circ} \mathrm{C}$ (d). CRABP-RA complex ion peaks are marked with a filled square, apo-CRABP ion peaks are marked with an open square.

1:1 to 5:1 (RA:CRABP), the proportion of holo-protein ions increased in the ESI spectrum, while the binding stoichiometry remained 1:1 (data not shown). It appears that the proportion of complex present in the ESI spectra depends not only on ESI conditions but also on the relative concentration of ligand. When the molar ratio of RA:CRABP is 5:1, we were able to observe the spectrum with $98 \%$ ligand-bound complex ions under mild source conditions. In all further experiments, therefore, the ligand:protein molar ratio was maintained at 5:1 to insure complete formation of the holoprotein.

The correlation between the proportion of the complex present in ESI spectra and the relative concentrations of protein and ligand in solution often suggests the possibility of non-specific protein-ligand interactions [13]. However, non-specific interactions in the gas phase are also likely to alter the observed stoichiometry of the complex ion in a concentration-dependent manner, a phenomenon not observed in the ATRA-CRABP I mixtures presented here (vide infra).

ESI MS spectra of CRABP I in the presence of RA isomers and retinol acquired at neutral $\mathrm{pH}$ are shown in Figure 3. The proportion of the ligand-bound forms of CRABP I (1:1 stoichiometry) is significant, although not as high as the ATRA-CRABP I complex, as judged by relative intensities of holo- and apo-protein ion intensities in the ESI spectra (Figure 3b, c). Alteration of the 


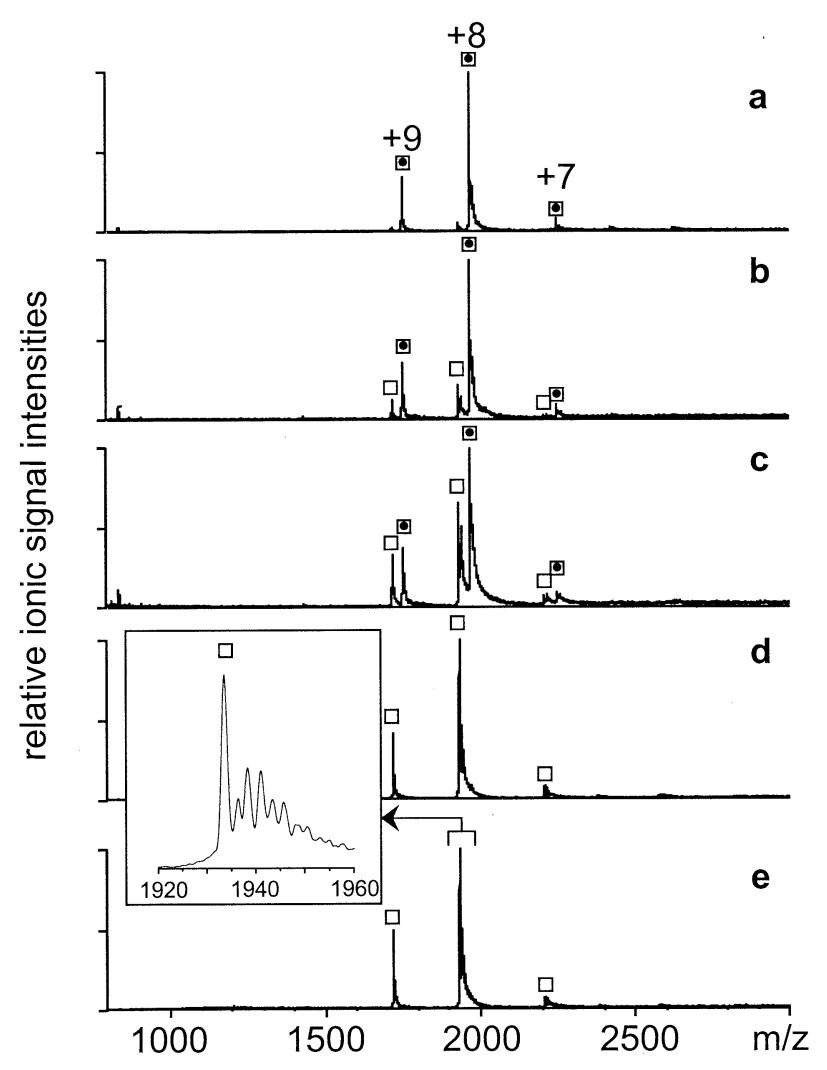

Figure 3. ESI mass spectra of CRABP I mixtures with RA isomers (a) retinoic acid, (b) 13-cis-RA, (c) 9-cis-RA, (d) retinol, and (e) in the absence of RA isomers. All spectra were collected under the following conditions: $\mathrm{V}_{\mathrm{O}-\mathrm{L}}=60 \mathrm{~V}, \mathrm{~T}_{\mathrm{D}}=60^{\circ} \mathrm{C}$. The inset is the enlargement of the intact ion peak at +8 charge state, a variety of adducts including sodium, potassium and acetate are observed.

hydrophobic backbone in 9-cis RA and 13-cis RA disrupts the hydrophobic protein-ligand contacts, while the electrostatic portion is not affected. This weakens the overall interaction, a fact confirmed by fluorimetric titration [22]. Our measurements are consistent with this notion, suggesting the following order of relative affinities: ATRA > 13-cis-RA > 9-cis-RA. However, it is noted that the ligand binding affinities of the two isomers of retinoic acid to CRABP I are in the reversed order as compared the results of fluorimetric studies: ATRA $>$ 9-cis-RA $>$ 13-cis-RA [22, 29]. This suggests that the protein-ligand complex stability in the gas phase does not necessarily mirror the binding strength in solution, particularly if hydrophobic interactions play an important role. Complete elimination of the hydrophobic skeleton of RA gives rise to non-specific protein-ligand interactions, as evidenced by a range of acetate adducts with varying stoichiometry present in all ESI spectra acquired under mild conditions (Figure 3 inset).

A control ESI spectrum of CRABP I in the presence of a five-fold molar excess of retinol $(\mathrm{ROH})$ has been obtained under the same ESI interface conditions as the ATRA-CRABP I mixture. ROH has the same hydrophobic backbone as ATRA, but lacks a terminal carboxylate group (Figure 1), which effectively eliminates binding to CRABP I [23]. The spectrum of the ROH-CRABP I mixture contains only protein ion peaks corresponding to the apo-form of the protein (Figure 3d). However, this observation should be approached with some skepticism, as one might argue that even if the $\mathrm{ROH}-\mathrm{CRABP}$ I complex were formed in solution, it may not survive the transition to the gas phase due to the absence of any electrostatic interactions between the complex constituents. Clearly, one needs to devise an alternative strategy in order to prove absence of binding in solution.

\section{Protein Dynamics and Ligand Binding-HDX Measurements at Neutral $p H$}

Recent progress in the areas of protein folding and binding has led to the realization that the two processes should not necessarily be considered separately from each other [30]. This notion is often used when studying protein-protein interactions [31, 32], however, it can also be very useful when protein interaction with small organic ligands is considered. A common assumption here is that binding reduces flexibility [33,34], which has been proven experimentally in the case of several retinoid carriers [19, 35, 36]. Therefore, changes in protein backbone dynamics upon ligand introduction can be used as indicators of the protein-ligand interaction. To test this hypothesis, we measured HDX kinetics of CRABP under near-native conditions $(\mathrm{pH} \mathrm{7,10} \mathrm{mM}$ $\mathrm{CH}_{3} \mathrm{CO}_{2} \mathrm{NH}_{4}$ ) both in the absence and in the presence of a range of retinoids (ATRA, 9-cis-RA, 13-cis-RA, and $\mathrm{ROH})$, Figures 4 and 5 . The experiment was conducted at $36^{\circ} \mathrm{C}$ in order to reduce the HDX measurement time compared to room temperature.

Hydrogen exchange of protons in folded proteins in solution depends on two processes, one being the unfolding event which exposes the amide to solvent, and the other the intrinsic exchange of an exposed amide with bulk solvent [37]. Under most conditions (including physiological conditions), protein refolding rates are much higher than the intrinsic amide exchange rates (a situation commonly known as the $\mathrm{EX}_{2}$ exchange regime) [2]. The observed rate equation is

$$
k_{\text {obs }}=K_{o p} \cdot k_{\text {int }}
$$

where $\mathrm{K}_{\mathrm{op}}$ is the unfolding equilibrium constant and $k_{\mathrm{int}}$ is the intrinsic amide exchange rate constant. This gives rise to a non-uniform incorporation of deuterium across the population of protein molecules in solution. As a consequence, the observed mass spectra will contain a single peak, gradually shifting in mass as the exchange time increases.

HDX of CRABP I at pH 7 clearly follows the $\mathrm{EX}_{2}$ exchange mechanism (Figure 4). As expected, the measured HDX kinetics change dramatically upon introduction of ATRA to the exchange buffer. After $60 \mathrm{~min}$ of exchange, only $34 \pm 2$ protected deuterons remain in the apo-form of the protein, whereas in the presence of 


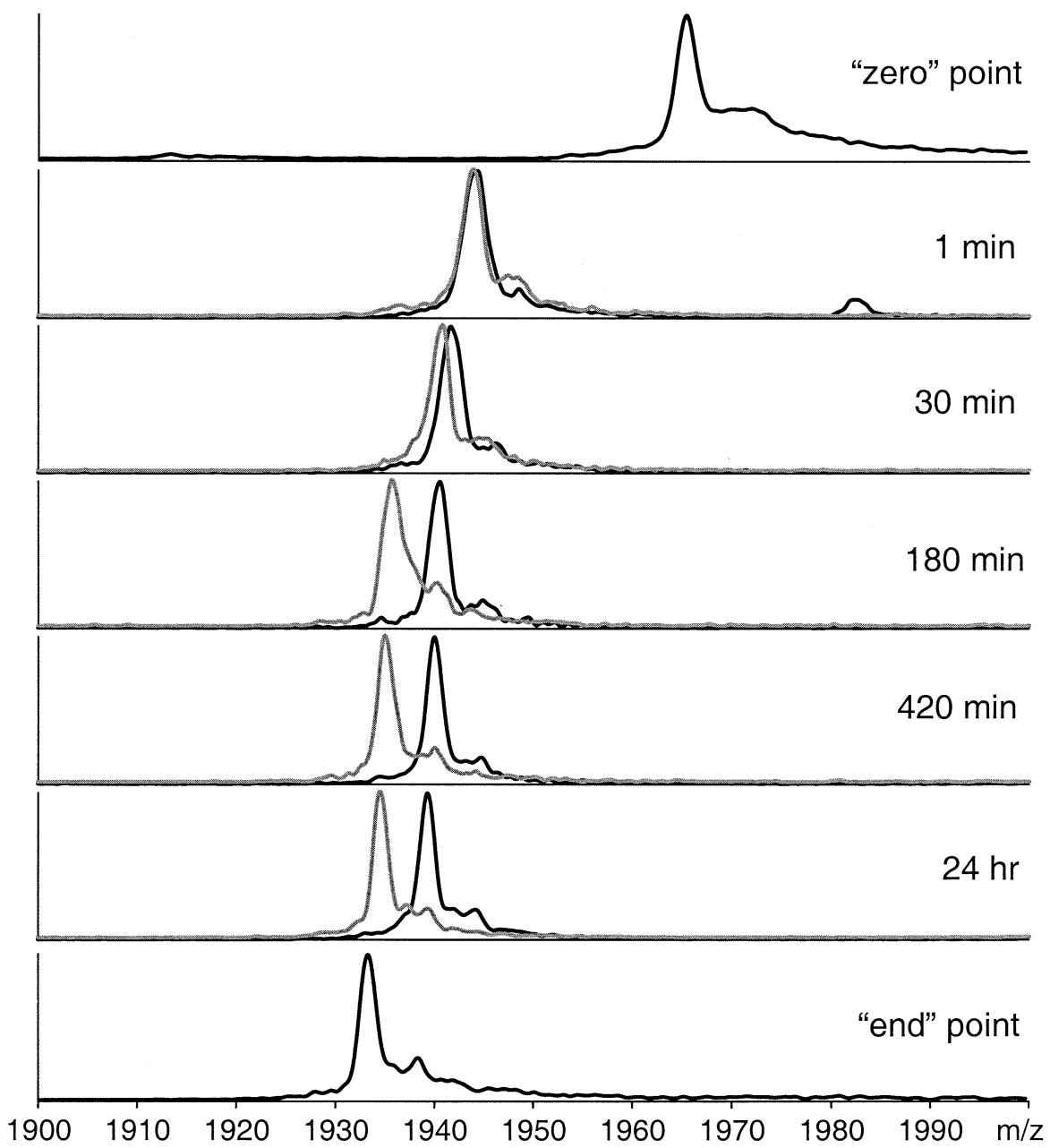

Figure 4. Evolution of a peak shape of a +8 ion of CRABP I undergoing HDX in the absence (grey line) and in the presence (black line) of five-fold molar excess of ATRA under "near-native" conditions

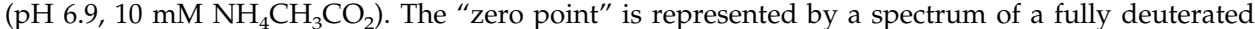
protein in $\mathrm{D}_{2} \mathrm{O} / \mathrm{CD}_{3} \mathrm{CO}_{2} \mathrm{ND}_{4}$. The "end point" of the exchange is represented by a spectrum of unlabeled CRABP I in protiated solution. Protein concentration is $1.2 \mu \mathrm{M}$, protein/ligand molar ratio is 1:5. ESI source settings: $\mathrm{V}_{\mathrm{O}-\mathrm{L}}=120 \mathrm{~V}, \mathrm{~T}_{\mathrm{D}}=80^{\circ} \mathrm{C}$.

ATRA, some $56 \pm 2$ amides remain protected. Importantly, the HDX kinetics in the ATRA-CRABP I system is not dependent on the ATRA concentration, so long as molar concentration of the ligand is equal to or exceeds that of the protein, sufficient to insure saturated binding. The fraction of protected amides as a function of the exchange time was measured in a 1:1 ATRACRABP I mixture and was found to be nearly identical to that measured in the 5:1 mixture (data not shown).

The other isomers of ATRA do not afford such significant protection to CRABP I, resulting in only $11 \pm$ 2 (9-cis RA) and $8 \pm 2$ (13-cis $>$ RA) more amides sequestered from solvent after $1 \mathrm{~h}$ of exchange compared to the apo-form of the protein. It is clear, therefore, that the presence of both 9- and 13-cis isomers does reduce the backbone mobility, although not to such a significant extent as the physiological ligand ATRA. HDX of CRABP in the presence of $\mathrm{ROH}$ is almost identical to the apo-form, consistent with the notion that $\mathrm{ROH}$ does not bind to CRABP I. This also demonstrates that protection does not result simply from non-specific hydrophobic interactions in solution. Thus, HDX measurements at neutral $\mathrm{pH}$ provide correct ranking of the retinoid affinities to CRABP I: ATRA $\gg$ 9-cis $\mathrm{RA}>13$-cis-RA $\gg$ retinol (no binding).

To determine the rate constants $k_{\mathrm{obs}}$, the experimentally measured curves representing amide protection as a function of exchange time were fitted using a multimodal exponential decay:

$$
N(t)=\sum_{i=1}^{n-1} A_{i} \cdot e^{-k_{i} t}
$$

The following restrictions were applied: (1) a summation of pre-exponential factors was set to 107 and (2) the set of pre-exponential factors $\mathrm{A}_{i}$ was the same for both apo- and holo-forms of the protein. The first restriction (1) above reflects the fact that there are 107 backbone amide hydrogen atoms protected (H-bonded) in the 


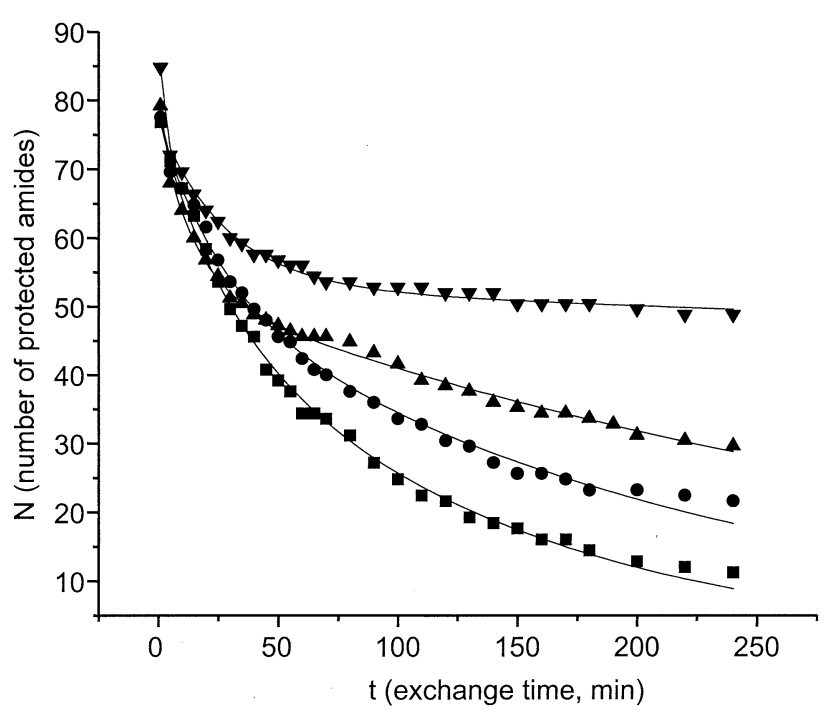

Figure 5. H/D exchange kinetics of CRABP I in the presence of ATRA (inverted filled triangle), 9-cis-RA (filled triangle), 13-cis-RA (filled circle), and in the absence of the ligand (filled square). Protein concentration $1.2 \mu \mathrm{M}$ (in $10 \mathrm{mM} \mathrm{NH}_{4} \mathrm{CH}_{3} \mathrm{CO}_{2}, \mathrm{pH}$ 6.9). Protein:ligand molar ratio is 1:5. ESI source settings: $\mathrm{V}_{\mathrm{O}-\mathrm{L}}=120 \mathrm{~V}$, $\mathrm{T}_{\mathrm{D}}=80^{\circ} \mathrm{C}(\mathbf{a})$. Each curve was fitted with triple-modal exponential decay function $\mathrm{N}=30 \exp \left(-k_{1} \cdot \mathrm{t}\right)+24 \exp \left(-k_{2} \cdot \mathrm{t}\right)+53$ $\exp \left(-k_{3} \cdot \mathrm{t}\right)$ (calculated $k$ values are shown in Table 1$)$.

native state of both apo- and holo- forms of CRABP I according to available crystal structures $[15,38]$. This can be demonstrated using ESI-MS by allowing exchange to occur under conditions where the intrinsic amide exchange rate is significantly lower and hence an initial time point can be measured more readily. At $\mathrm{pH}$ 3.5 and $20^{\circ} \mathrm{C}$, the average intrinsic exchange rate is reduced to as low as $0.005 \mathrm{~s}^{-1}$, with a corresponding half-life of about $2.5 \mathrm{~min}$ [39]. Our experiments conducted at $\mathrm{pH} 3.5$ under room temperature show in all cases the $2 \mathrm{~min}$ exchange time point for CRABP I is identical regardless the presence of ligands, with $107 \pm$ 3 amides protected, justifying the first restriction above (data not shown).

The second restriction reflects an implicit assumption that there are a total of $n$ different states of the protein, including the native state, random coil and $n-2$ intermediate states whose structures (but not the energetics) are unaffected by the presence of ligand. The total number of protein states participating in HDX (n) was determined by deconvolution of charge state distributions [5]. The same number of states are observed with the same basis sets regardless of apo-/holo- and ligand type. This implies that "activated" states, which may be important for the ligand-binding process, are similar in apo- and holo-forms and that non-cognate ligands do not alter the native state structure. This implies that dissociation of the ligand from the protein occurs through the same mechanism as binding (although in reverse order). Although this assumption appears to be reasonable, its validity will be tested in a later section of this paper.

Curve fitting of the experimental data for the apoand holo-forms of the protein (in complex with ATRA) produced satisfactory results (as judged by randomness of residuals) only when a tri-exponential decay was used to model the HDX kinetics (see Table 1). This suggests that the total number of states is four (implying two intermediate states can become transiently populated under native conditions). Figure 6 depicts hypothetical energy landscapes of the protein with and without the ligand. The fast phase of the exchange represents transient formation of a highly structured intermediate state $\left(\mathbf{I}_{1}, 32\right.$ fewer protected amides compared to the native state), while the medium phase represents a transient formation of a less structured state $\left(\mathbf{I}_{2}, 56\right.$ fewer protected amides compared to the native state). The slowest HDX phase represents transient global unfolding of the protein $(\mathbf{U}$, i.e., formation of a random coil state with little or no protection). Interestingly, when HDX of CRABP I (apo-form) was monitored by NMR, only 71 protected amides were detected (S.J.E. and L.M. Gierasch, unpublished data). Our measurements suggest that the transition from the native state to the highly structured intermediate $\left(\mathrm{N} \rightarrow \mathrm{I}_{1}\right.$ ) occurs on the time scale of less than $2 \mathrm{~min}$ at $36{ }^{\circ} \mathrm{C}\left(5 \mathrm{~min}\right.$ at $\left.25^{\circ} \mathrm{C}\right)$ and thus these amides would exchange too rapidly to be detected by NMR. According to our measurements, this transition accounts for a loss of protection at 30 amide hydrogen atoms, thus leaving only 77 amides that remain protected on a time scale readily accessible to 2-D HDX/NMR (>30 min).

Assuming that specific binding is the only factor responsible for modulation of the backbone dynamics, and that there is no ligand interaction between the ligand and the fully unstructured protein, we can provide estimates of the binding energy based on the measured HDX kinetics. The protein free energy gain upon unfolding from the native state $\Delta G$ relates to the unfolding equilibrium constant in a two-state process simply as

Table 1. HDX rates of CRABP I at $\mathrm{pH} 7\left(36^{\circ} \mathrm{C}\right)^{\mathrm{a}}$

\begin{tabular}{lccc}
\hline Ligand & Fast phase $\left(\mathrm{min}^{-1}\right)$ & Medium phase $\left(\mathrm{min}^{-1}\right)$ & Slow phase $\left(\mathrm{min}^{-1}\right)$ \\
\hline \hline None & $3.29 \pm 1.00$ & $0.037 \pm 0.002$ & $0.0074 \pm 0.0001$ \\
13-cis RA & $2.83 \pm 0.63$ & $0.037 \pm 0.002$ & $0.0044 \pm 0.001$ \\
9-cis RA & $2.02 \pm 0.27$ & $0.068 \pm 0.006$ & $0.0025 \pm 0.001$ \\
ATRA & $1.24 \pm 0.12$ & $0.035 \pm 0.002$ & $0.00026 \pm 0.00008$ \\
\hline
\end{tabular}

${ }^{a}$ All the errors are from curve fitting only. 

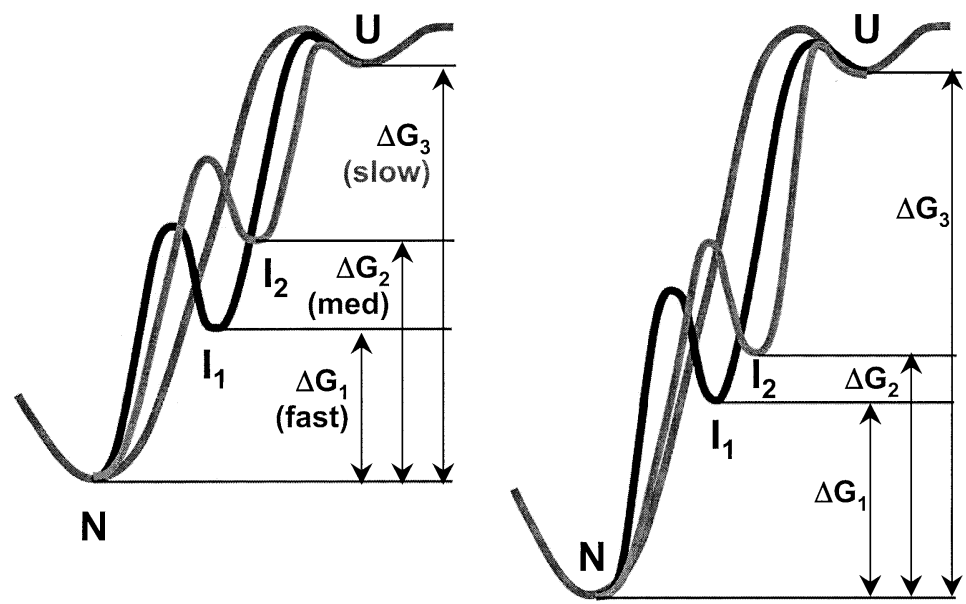

Figure 6. A diagram of hypothetical energy landscapes of CRABP I in the apo- (left) and holo- (right) forms. Both intermediate states and the native state are stabilized in the presence of the ligand.

$$
\Delta G=-R T \cdot \ln \left(K_{o p}\right)
$$

Therefore, one can use the values of $\mathrm{K}_{\mathrm{op}}$ (measured in the absence and in the presence of ATRA) to determine the change in the protein stability $\Delta \Delta \mathrm{G}$ upon introduction of the ligand into the protein solution, which should be numerically equal to the protein-ligand binding energy. The equilibrium constants $\mathrm{K}_{\mathrm{op}}$ can be calculated based on the measured HDX kinetics; the difference in stability of the native conformation can be calculated as

$$
\Delta \Delta G=R T \cdot \ln \left(\frac{K_{o p}^{a p o}}{K_{o p}^{\text {holo }}}\right)
$$

Calculation of $\Delta \Delta \mathrm{G}_{3}$, the free energy difference derived from the slowest exchange process, based on the experimental data (Table 1) gives $2 \mathrm{kcal} / \mathrm{mol}$ as an estimate of the ATRA-CRABP I binding energy. The calculation is reasonably close to the stabilization energy measured calorimetrically, considering the possibility of protein aggregation under conditions employed in a typical DSC experiment [16].

The intermediate states are also stabilized in the presence of the ligand (see Table 2 and Figure 6). Thus, $\Delta \Delta \mathrm{G}_{1}=0.6 \mathrm{kcal} / \mathrm{mol}$, indicating that the free energy of the more structured intermediate $\left(\mathbf{I}_{1}\right)$ decreases by 1.4 $\mathrm{kcal} / \mathrm{mol}$ in the presence of ATRA. Interestingly, the less structured intermediate species $\left(\mathbf{I}_{2}\right)$ appears to be stabilized in the presence of ATRA by $2 \mathrm{kcal} / \mathrm{mol}$ (since the calculated $\Delta \Delta \mathrm{G}_{2}=0.03 \mathrm{kcal} / \mathrm{mol}$ is close to thermal energy).

Thermodynamics of 9-cis and 13-cis RA interaction with CRABP I based on HDX measurements suggest that CRABP I is stabilized by these two retinoids although to a lesser extent compared to the cognate ligand all-trans RA: $\Delta \Delta \mathrm{G}_{3}=0.6 \mathrm{kcal} / \mathrm{mol}$ for $9-c i s$, and $0.3 \mathrm{kcal} / \mathrm{mol}$ for 13 -cis RA.
Protein Dynamics and Ligand Binding Under Mildly Denaturing Conditions: Correlating Ligand Binding with Specific Dynamic Events

Characterization of the protein backbone flexibility modulation by ligand under native conditions provides the most straightforward way to probe binding events by measuring protein dynamics. However, it is important to remember that estimation of $\Delta \Delta G^{\text {binding }}$ simply as $\Delta \Delta \mathrm{G}$ (based on the measured values of $\mathrm{K}_{\mathrm{op}}$ ) relies on an implicit assumption that the conformational changes upon binding are negligible, i.e., the structure of apoand holo-forms are identical. As illustrated in our hypothetical energy landscape of apo- and holo-form of the protein (Figure 6), favorable ligand-protein interactions decrease the free energy of the native state of protein upon ligand binding, without affecting the native state conformation. Furthermore, the second restriction of $\mathrm{Eq} 1$ above relies on the assumption that any structured intermediates which are important for ligand binding and release remain the same in nature regardless of the ligand, i.e., a dynamic "lock-and-key" binding mechanism as opposed to an "induced fit". We postulate that ligand-binding may affect the free energy of the intermediate states without altering their structures. The free energy of each intermediate should be decreased to a lesser extent upon ligand binding (compared to the $\Delta \Delta \mathrm{G}$ of the native state), since they have less favorable ligand-protein interactions. The crystal structures of the ligand-free and bound forms of the

Table 2. Analysis of HDX curves for CRABP I in the absence and presence of ligands

\begin{tabular}{lccc}
\hline & $\begin{array}{c}\Delta \Delta \mathrm{G}_{1} \\
(\mathrm{kcal} / \mathrm{mol})\end{array}$ & $\begin{array}{c}\Delta \Delta \mathrm{G}_{2} \\
(\mathrm{kcal} / \mathrm{mol})\end{array}$ & $\begin{array}{c}\Delta \Delta \mathrm{G}_{3} \\
(\mathrm{kcal} / \mathrm{mol})\end{array}$ \\
\hline \hline 13-cis RA & 0.09 & - & 0.3 \\
9-cis RA & 0.3 & - & 0.6 \\
ATRA & 0.6 & 0.03 & 2 \\
\hline
\end{tabular}




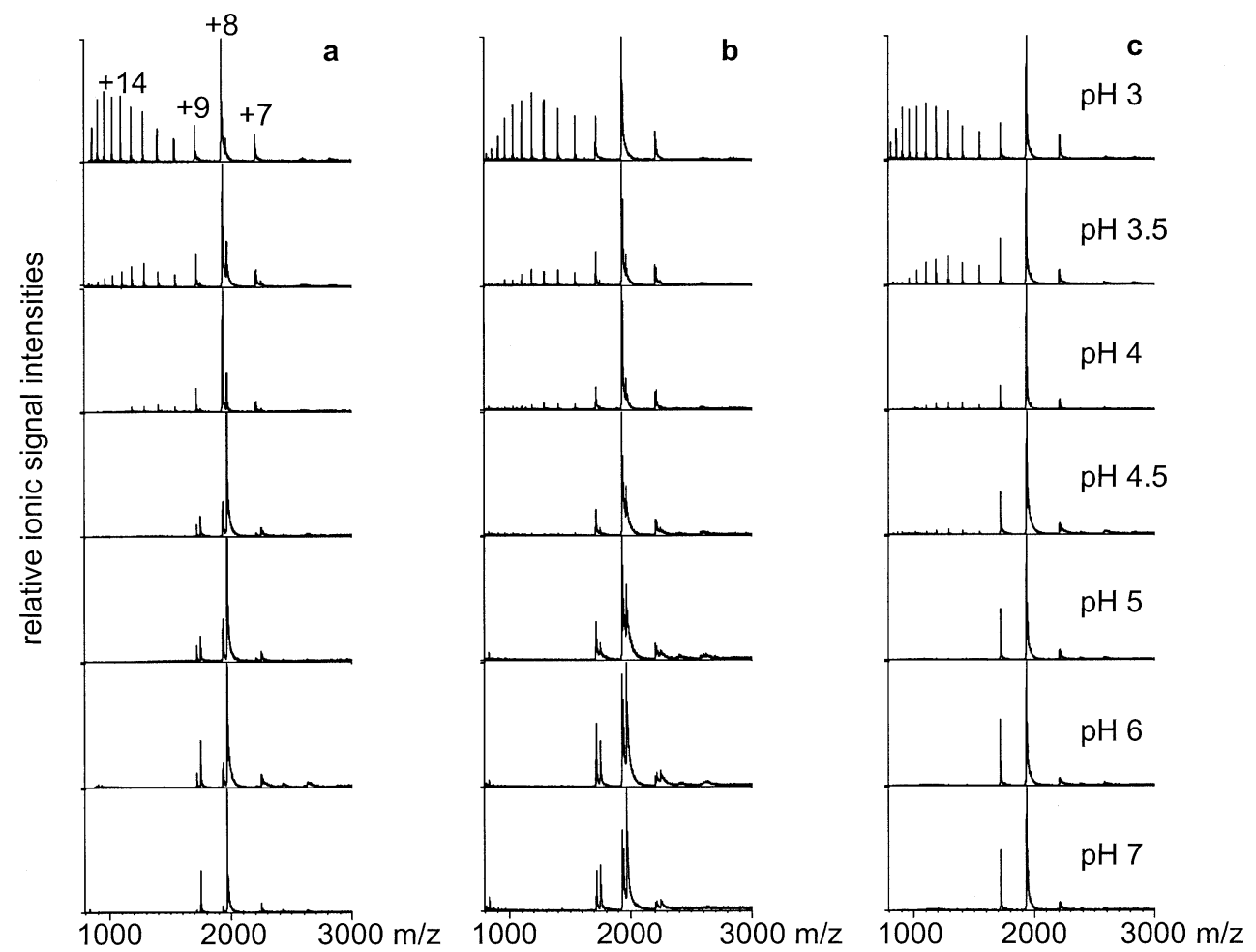

Figure 7. Charge state distribution of CRABP I with and without ligand: ATRA (a), 9-cis-RA-CRABP (b), and apo-protein (c). ESI interface conditions: $\mathrm{V}_{\mathrm{O}-\mathrm{L}}=60 \mathrm{~V}, \mathrm{~T}_{\mathrm{D}}=60^{\circ} \mathrm{C}$.

native state are effectively identical [15], but there is no direct evidence to demonstrate the similarity of transient partially folded conformers. In fact, one might argue that accommodation of RA isomers within the binding cavity of CRABP I may induce significant local conformational alterations. Therefore, in order to validate the measurements presented in the previous section, one needs to prove that ligand-binding (ATRA or its isomers) does not induce any conformational changes within the protein, but simply shifts the equilibria among the conformers already present in the apo-form of the protein. We have demonstrated previously that the distribution of protein ion charge states in ESI-MS under native and mildly denaturing conditions can give clear indications as to the number and nature of the states present in solution [5]. Thus, we undertook a detailed analysis of the charge state distributions exhibited by CRABP I ions in the apo-form and in the presence of the various ligands.

As shown in Figure 7, the spectrum of CRABP I at neutral $\mathrm{pH}$ shows a very narrow distribution of charge states (ranging from +7 to +9 ). A distribution with higher charge states (low $\mathrm{m} / \mathrm{z}$ ) appears at lower $\mathrm{pH}$, owing to the ability of unfolded protein molecules to accommodate charges more readily than the folded protein. Importantly, the protein-ligand complex ions become significantly less abundant below $\mathrm{pH} 5$, and yet the ATRA-CRABP I complex ions can be observed as low as $\mathrm{pH}$ 3. It is notable that only ion peaks of lower charge states (from +7 to +9 ) contain the proteinligand complex form, whereas only ion peaks corre- sponding to the ligand-free form were observed in the higher charge states. Interestingly, the spectra contain no evidence that the binding-competent intermediate states of the protein $\left(I_{1}\right.$ and $\left.I_{2}\right)$ form stable complexes with the ligand, although the HDX measurements carried out under native conditions indicate some stabilization of the two intermediate states induced by ligand binding. This suggests that these complexes are too unstable to be observed directly under the ESI source conditions employed here.

Onset of protein unfolding is observed in the presence of ligand between $\mathrm{pH} 4.5$ and 4 . At $\mathrm{pH} 4.5$ and above, the high charge density protein ions are absent from the mass spectra of CRABP I in the presence of retinoids. In the apo-form, however, this unfolded distribution persists, providing further evidence that ligand binding stabilizes the protein by effectively inhibiting sampling of the more unfolded ensembles. Importantly, the order of binding preferences of retinoids to the protein's native conformation remains unchanged throughout the entire $\mathrm{pH}$ range studied.

The fraction of ligand-bound protein ions is plotted versus $\mathrm{pH}$ in Figure 8, where the relative intensities of the protein-ligand complex ions were monitored as a function of $\mathrm{pH}$. The peak intensities for the most abundant ion peaks $(+8)$ of the apo-protein and protein-ligand complex were compared to determine the fraction of protein-ligand complex ion at each $\mathrm{pH}$. There is a sharp decrease in the intensity of the ligandbound protein ions at $\mathrm{pH}<4.5$, with the midpoint of the transition occurring at about $\mathrm{pH} 4$. The onset of 


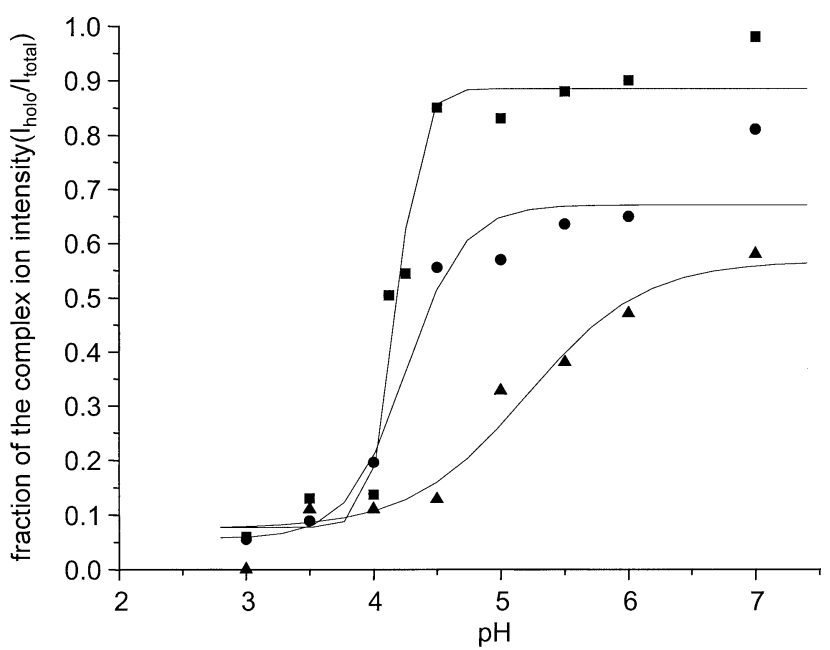

Figure 8. ESI MS titration plot, monitoring the fraction of CRABP I-retinoid complex with increasing $\mathrm{pH}$ : ATRA (filled square), 13-cis-RA (filled circle), and 9-cis-RA (filled triangle).

unfolding of the protein in the ATRA-CRABP I mixture, on the other hand, occurs only below $\mathrm{pH} 4$. It is probable that disappearance of the holo-form from the spectra results from RA protonation if its $\mathrm{p} K_{\mathrm{a}}$ is within this $\mathrm{pH}$ range (the isomeric form isotretinoin has a $\mathrm{pK}$ of 3.8 [40]), followed by protein denaturation as the $\mathrm{pH}$ is further reduced. The less cooperative nature of the binding curve for the CRABP-9-cis-RA (and to a lesser extent 13-cis-RA) complex suggests relatively weak binding between protein and 9-cis or 13-cis RA relative to the all-trans form.

\section{Conclusions}

In this paper we have demonstrated that additional protection against amide hydrogen exchange afforded by binding of small hydrophobic ligands to CRABP I may be used to determine the efficacy of ligand-binding, thus enabling an order of binding preference to be measured. Retinoic acid does not form hydrogen bonds to the peptide backbone of the protein, hence all "ligand-induced" protection can be attributed to reduced protein flexibility. By comparing the protein hydrogen exchange behaviors in the presence of ligands with gradually reduced binding affinities, we are able to determine the ligand-binding effect on protein conformational dynamics. Protection against exchange of CRABP I in the presence of 13-cis- and 9-cis-RA is lower relative to that in the presence of all-trans-RA, consistent with the order of binding affinity to the protein. The binding preference of retinoids to CRABP I has the order of alltrans-RA > 9-cis-RA > 13-cis-RA, as determined by our HDX/MS measurements, which is in agreement with the solution-based fluorimetric measurements. This method is more accurate than traditional direct ESI MS measurements and may be used to extract the protein-ligand binding energy. In the presence of retinol, the protein is no more protected against exchange than the apo-form itself, thus demonstrating that the protein neither binds this ligand specifically, nor is exchange affected by non-specific interactions.

The exchange kinetics exhibit multiple rate constants, indicating that the exchange occurs not only upon complete unfolding of the protein, but also a number of partially folded "activated" states are involved in this process. Exchange from these states is also affected by the presence of RA, suggesting that the ligand is also associated with these conformers. A detailed analysis of the charge state distributions under native and mildly denaturing conditions demonstrates that these activated states are populated to varying extents under all conditions. We postulate that these conformers are important mechanistically for the ligand-binding process, since the native state itself (as judged from the crystal structure) does not have an opening sufficient to allow ligand access to the binding cavity [38].

By performing exchange experiments on CRABP I in the presence of ligand but under mildly denaturing conditions, we can increase the Boltzmann weight of these partially folded conformers and, using HDX ESI-MS under conditions favoring the $\mathrm{EX}_{1}$ regime, we can measure protection in these individual states [20]. This unique capability of mass spectrometry to distinguish protection in each state will shed light on the dynamic processes associated with ligand binding. In combination with fragmentation methods and FT ICR MS [21], we also plan to determine site-specifically the regions of the protein important for ligand-binding specificity.

\section{Acknowledgments}

The authors thank Professor Lila M. Gierasch (University of Massachusetts, Department of Biochemistry and Molecular Biology) for providing CRABP I samples. This work was supported by a grant from the National Institutes of Health (R01 GM61666).

\section{References}

1. Loo, J. A. Studying Non-covalent Protein Complexes by Electrospray Ionization Mass Spectrometry. Mass Spectrom. Rev. 1997, 16, 1-23.

2. Engen, J. R.; Smith, D. L. Investigating Protein Structure and Dynamics by Hydrogen Exchange MS. Anal. Chem. 2001, 73, 256A-265A.

3. Kaltashov, I. A.; Eyles, S. J. Studies of Biomolecular Conformations and Conformational Dynamics by Mass Spectrometry. Mass Spectrom. Rev. 2002, 21, 37-71.

4. Konermann, L.; Douglas, D. J. Equilibrium Unfolding of Proteins Monitored by Electrospray Ionization Mass Spectrometry: Distinguishing Two-State from Multi-State Transitions. Rapid Commun. Mass Spectrom. 1998, 12, 435-442.

5. Dobo, A.; Kaltashov, I. A. Detection of Multiple Protein Conformational Ensembles in Solution via Deconvolution of Charge-State Distributions in ESI MS. Anal. Chem. 2001, 73, 4763-4773. 
6. Gumerov, D. R.; Kaltashov, I. A. Dynamics of Iron Release from Transferrin N-Lobe Studied by Electrospray Ionization Mass Spectrometry. Anal. Chem. 2001, 73, 2565-2570.

7. Kolakowski, B. M.; Konermann, L. From Small-Molecule Reactions to Protein Folding: Studying Biochemical Kinetics by Stopped-Flow Electrospray Mass Spectrometry. Anal. Biochem. 2001, 292, 107-114.

8. Simmons, D. A.; Konermann, L. Characterization of Transient Protein Folding Intermediates During Myoglobin Reconstitution by Time-Resolved Electrospray Mass Spectrometry with On-Line Isotopic Pulse Labeling. Biochemistry 2002, 41, 19061914.

9. Wine, R. N.; Dial, J. M.; Tomer, K. B.; Borchers, C. H. Identification of Components of Protein Complexes Using a Fluorescent Photo-Cross-Linker and Mass Spectrometry. Anal. Chem. 2002, 74, 1939-1945.

10. Englander, S. W.; Mayne, L.; Bai, Y.; Sosnick, T. R. Hydrogen Exchange: The Modern Legacy of Linderstrom-Lang. Protein Sci. 1997, 6, 1101-1109.

11. Mandell, J. G.; Falick, A. M.; Komives, E. A. Identification of Protein-Protein Interfaces by Decreased Amide Proton Solvent Accessibility. Proc. Natl. Acad. Sci. U.S.A 1998, 95, 1470514710.

12. Jarrold, M. F. Peptides and Proteins in the Vapor Phase. Annu. Rev. Phys. Chem. 2000, 51, 179-207.

13. Robinson, C. V.; Chung, E. W.; Kragelund, B. B.; Knudsen, J.; Aplin, R. T.; Poulsen, F. M.; Dobson, C. M. Probing the Nature of Non-Covalent Interactions by Mass Spectrometry. A study of Protein-CoA Ligand Binding and Assembly. J. Am. Chem. Soc. 1996, 118, 8646-8653.

14. Donovan, M.; Olofsson, B.; Gustafson, A. L.; Dencker, L.; Eriksson, U. The Cellular Retinoic Acid Binding Proteins. J. Steroid Biochem. Mol. Biol. 1995, 53, 459-465.

15. Kleywegt, G. J.; Bergfors, T.; Senn, H.; Le Motte, P.; Gsell, B.; Shudo, K.; Jones, T. A. Crystal Structures of Cellular Retinoic Acid Binding Proteins I and II in Complex with All-trans-Retinoic Acid and a Synthetic Retinoid. Structure 1994, 2, 1241-1258.

16. Zhang, J.; Liu, Z. P.; Jones, T. A.; Gierasch, L. M.; Sambrook, J. F. Mutating the Charged Residues in the Binding Pocket of Cellular Retinoic Acid-Binding Protein Simultaneously Reduces Its Binding Affinity to Retinoic Acid and Increases Its Thermostability. Proteins 1992, 13, 87-99.

17. Sacchettini, J. C.; Gordon, J. I.; Banaszak, L. J. Crystal Structure of Rat Intestinal Fatty-Acid-Binding Protein. Refinement and Analysis of the Escherichia coli-Derived Protein with Bound Palmitate. J. Mol. Biol. 1989, 208, 327-339.

18. Winter, N. S.; Bratt, J. M.; Banaszak, L. J. Crystal Structures of Holo- and Apo-Cellular Retinol-Binding Protein II. J. Mol. Biol. 1993, 230, 1247-1259.

19. Krishnan, V. V.; Sukumar, M.; Gierasch, L. M.; Cosman, M. Dynamics of Cellular Retinoic Acid Binding Protein I on Multiple Time Scales with Implications for Ligand Binding. Biochemistry 2000, 39, 9119-9129.

20. Eyles, S. J.; Dresch, T.; Gierasch, L. M.; Kaltashov, I. A. Unfolding Dynamics of a $\beta$-Sheet Protein Studied by Mass Spectrometry. J. Mass Spectrom. 1999, 34, 1289-1295.

21. Eyles, S. J.; Speir, J. P.; Kruppa, G. H.; Gierasch, L. M.; Kaltashov, I. A. Protein Conformational Stability Probed by Fourier Transform Ion Cyclotron Resonance Mass Spectrometry. J. Am. Chem. Soc. 2000, 122, 495-500.

22. Norris, A. W.; Cheng, L.; Giguere, V.; Rosenberger, M.; Li, E. Measurement of Subnanomolar Retinoic Acid Binding Affinities for Cellular Retinoic Acid Binding Proteins by Fluorometric Titration. Biochim. Biophys. Acta 1994, 1209, 10-18.
23. Ong, D. E.; Chytil, F. Retinoic Acid-Binding Protein in Rat Tissue. Partial Purification and Comparison to Rat Tissue Retinol-Binding Protein. J. Biol. Chem. 1975, 250, 6113-6117.

24. Chaudhuri, B. N.; Kleywegt, G. J.; Broutin-L'Hermite, I.; Bergfors, T.; Senn, H.; Le Motte, P.; Partouche, O.; Jones, T. A. Structures of Cellular Retinoic Acid Binding Proteins I and II in Complex with Synthetic Retinoids. Acta Crystallogr. D Biol. Crystallogr. 1999, 55, 1850-1857.

25. Thacher, S. M.; Vasudevan, J.; Chandraratna, R. A. Therapeutic Applications for Ligands of Retinoid Receptors. Curr. Pharm. Des. 2000, 6, 25-58.

26. Burns, L. L.; Dalessio, P. M.; Ropson, I. J. Folding Mechanism of Three Structurally Similar $\beta$-Sheet Proteins. Proteins 1998, 33, 107-118.

27. Cogan, U.; Kopelman, M.; Mokady, S.; Shinitzky, M. Binding Affinities of Retinol and Related Compounds to Retinol Binding Proteins. Eur. J. Biochem. 1976, 65, 71-78.

28. Wang, Y.; Chang, W. Y.; Prins, G. S.; van Breemen, R. B. Simultaneous Determination of All-trans, 9-cis, 13-cis Retinoic Acid and Retinol in Rat Prostate Using Liquid Chromatography-Mass Spectrometry. J. Mass Spectrom. 2001, 36, 882-888.

29. Fiorella, P. D.; Giguere, V.; Napoli, J. L. Expression of Cellular Retinoic Acid-Binding Protein (type II) in Escherichia coli. Characterization and Comparison to Cellular Retinoic AcidBinding Protein (Type I). J. Biol. Chem. 1993, 268, 21545-21552.

30. Baker, D.; Lim, W. A. From Folding Towards Function. Curr. Opin. Struct. Biol. 2002, 12, 11-13.

31. Tsai, C. J.; Kumar, S.; Ma, B.; Nussinov, R. Folding Funnels, Binding Funnels, and Protein Function. Protein Sci. 1999, 8, 1181-1190.

32. Verkhivker, G. M.; Bouzida, D.; Gehlhaar, D. K.; Rejto, P. A.; Freer, S. T.; Rose, P. W. Complexity and Simplicity of LigandMacromolecule Interactions: The Energy Landscape Perspective. Curr. Opin. Struct. Biol. 2002, 12, 197-203.

33. Sopkova, J.; Raguenes-Nicol, C.; Vincent, M.; Chevalier, A.; Lewit-Bentley, A.; Russo-Marie, F.; Gallay, J. $\mathrm{Ca}(2+)$ and Membrane Binding to Annexin 3 Modulate the Structure and Dynamics of Its N-Terminus and Domain III. Protein Sci. 2002, 11, 1613-1625.

34. Young, M. A.; Gonfloni, S.; Superti-Furga, G.; Roux, B.; Kuriyan, J. Dynamic Coupling Between the $\mathrm{SH} 2$ and $\mathrm{SH} 3$ Domains of c-Src and Hck Underlies Their Inactivation by C-Terminal Tyrosine Phosphorylation. Cell 2001, 105, 115-126.

35. Lu, J.; Lin, C. L.; Tang, C.; Ponder, J. W.; Kao, J. L.; Cistola, D. P.; Li, E. Binding of Retinol Induces Changes in Rat Cellular Retinol-Binding Protein II Conformation and Backbone Dynamics. J. Mol. Biol. 2000, 300, 619-632.

36. Franzoni, L.; Lucke, C.; Perez, C.; Cavazzini, D.; Rademacher, M.; Ludwig, C.; Spisni, A.; Rossi, G. L.; Ruterjans, H. Structure and Backbone Dynamics of Apo- and Holo-Cellular RetinolBinding Protein in Solution. J. Biol. Chem. 2002, 277, $21983-$ 21997.

37. Smith, D. L.; Deng, Y.; Zhang, Z. Probing the Non-Covalent Structure of Proteins by Amide Hydrogen Exchange and Mass Spectrometry. J. Mass Spectrom. 1997, 32, 135-146.

38. Thompson, J. R.; Bratt, J. M.; Banaszak, L. J. Crystal Structure of Cellular Retinoic Acid Binding Protein I Shows Increased Access to the Binding Cavity Due to Formation of an Intermolecular $\beta$-Sheet. J. Mol. Biol. 1995, 252, 433-446.

39. Connelly, G. P.; Bai, Y.; Jeng, M. F.; Englander, S. W. Isotope Effects in Peptide Group Hydrogen Exchange. Proteins 1993, 17, 87-92.

40. Walter, K.; Kurz, H. Binding of Drugs to Human Skin: Influencing Factors and the Role of Tissue Lipids. J. Pharm. Pharmacol. 1988, 40, 689-693. 